\title{
Measurement of internal and effective borehole resistances during thermal response tests
}

\author{
Louis Lamarche \\ J asmin Raymond \\ Claude Hugo Koubikana Pambou
}

\begin{abstract}
In a conventional thermal response test (TRT), the main parameter evaluated is the bulk subsurface thermal conductivity surrounding the borehole. It is also possible to evaluate the borehole thermal resistance. Several approaches were proposed in the literature to evaluate the possible combination of these two parameters. For example, it is often suggested to measure the temperature during the injection and the recovery periods, where the thermal conductivity is found with the recovery response whereas the borehole resistance is calculated with injection measurements. For this calculation, some authors suggested to use different means for the borehole temperature considering the asymmetric temperature distribution along the pipe legs that affects the borehole resistance. Some confusion about the borehole resistance that should be obtained may come from the difference between the 2D borehole resistance and the effective (3D) borehole resistance taking into account the internal heat transfer between the pipe legs inside the borehole. In practice, the latter one should be used in a design algorithm since it provides a more representative approach. In many cases, the difference between the two is rather small. However, since the borehole length is becoming an important variable to optimize, this difference in borehole resistance may represent a factor to better assess in the design of future systems. This effective resistance depends on the 2D borehole resistance, the water flow rate, the length of the borehole and the so called "internal resistance". To our knowledge, the in-situ assessment of this internal resistance has never been achieved. In this paper, we present our first investigation of a method that can be used to evaluate both the $2 \mathrm{D}$ borehole resistance $(\mathrm{R} b)$ and the $2 \mathrm{D}$ internal resistance (Ra). The method uses the temperature at the bottom of the borehole at the same time as the inlet and outlet temperatures that are measured in a conventional TRT. Interesting results were found by comparison with theoretical resistances calculated with the multipole method
\end{abstract}

\section{NTRODUCTI ON}

Conventional thermal response tests (TRTs), successfully implemented in the commercial geothermal sector, is to inject heat in a borehole and measure the temperature response from the heat pulse. Heat is normally generated by an electrical resistance outside the borehole and transported through a heat-transfer fluid, usually water flowing inside the borehole. Heat can also be generated using a heated cable inside the borehole (Raymond.et al. 2010 ). Heat pumps have alternatively been used with heated water circulating in a pilot ground heat exchanger (GHE). The method is mostly used to evaluate the subsurface thermal conductivity when designing ground-coupled heat pump (GCHP) systems but most of the time an evaluation of the borehole resistance is provided during the test. This last parameter is characteristic of the borehole heat transfer performances and can be assessed for quality control purposes

Louis Lamarche (louis.lamarche@etsmtl.ca) is a professor at École de Technologie Supérieure, Jasmin Raymond (jasmin.raymond@inrs.ca), is a professor at Institut national de la recherche scientifique, , Claude Hugo Koubikana Pambou is a doctoral student at Institut national de la recheche scientifique. 
(Raymond et al. 2014). Several methods have been proposed in the literature in order to evaluate these two parameters (Spitler and Gehlin 2015). The most common approach is to evaluate the thermal conductivity using the slope of the mean temperature increase with respect to the logarithm of the time. This formula comes from the well-known expression of the infinite line source (Carslaw and Jaeger 1959). Indeed, if we assume that the heat is released at the origin of the borehole and that it depends only of the radial conduction, which is a valid approximation for the time scale of a TRT test, the mean fluid temperature is given by:

$$
T_{f}(F o)-T_{o}=\frac{q_{i n j}^{\prime}}{4 \pi k_{s}} \int_{1 / 4 F o}^{\infty} \frac{e^{-u}}{u} d u=q_{i n j}^{\prime}(\overbrace{\frac{E_{1}(1 /(4 F o))}{4 \pi k_{s}}}^{R_{S}}+R_{b})
$$

Where $E_{1}$ is the exponential integral, $q_{i n j}^{\prime}$ is the amount of heat per meter injected during the test, $R_{b}$ is the borehole resistance and $\mathrm{Fo}$, the Fourier number based on the borehole radius. This expression is only valid for Fourier number larger than 5. For these values, it is known that the exponential integral is proportional to the natural logarithm of time. It follows that:

$$
k_{s}=\frac{q_{i n j}^{\prime}}{4 \pi m} \quad, \quad R_{b}=\frac{b-T_{O}}{q_{i n j}^{\prime}}-\frac{\ln \left(4 \alpha / r_{b}^{2}\right)-\gamma}{4 \pi k_{s}}
$$

where $\mathrm{m}$ is the slope and $\mathrm{b}$ is the intercept of the linear approximation (Fig. 1).

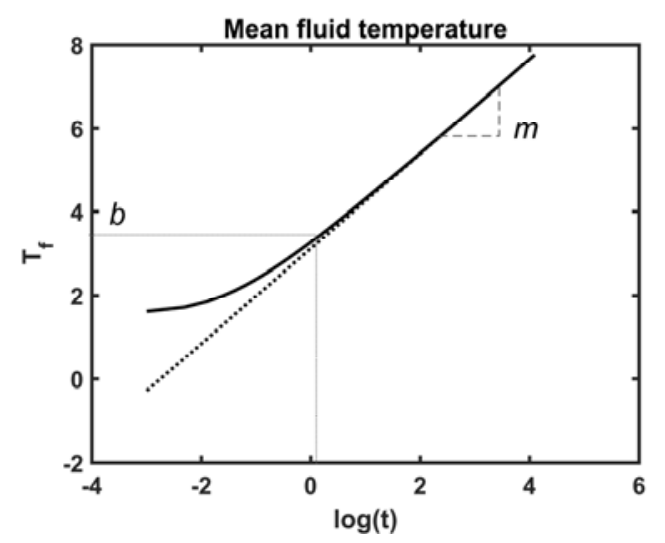

Figure 1 Classical slope method for the analysis of a TRT.

Some authors (Raymond et al. 2011) have suggested separating the TRT in two parts: an injection period that is done the same way as in the classical method followed by a thermal recovery period where no heat is injected. Since the temperatures become independent of the borehole resistance in during the thermal recovery, it is suggested to use this period to evaluate the thermal conductivity and the heat injection period to evaluate the borehole thermal resistance. Others researchers (Austin et al. 2000) used optimization methods to evaluate the unknown parameters using parameter estimation algorithm. Using this approach, other equations than the infinite line source function can 
be used for the thermal response factor. In all the previous expressions, $T_{f}$ represents the mean fluid temperature. Since most of the models neglect axial temperature variations, it is implicitly assumed that this mean temperature does not change with the depth and can be evaluated from the mean temperature at the exit of the borehole:

$$
T_{f}(t) \approx \frac{T_{f, \text { in }}+T_{f, \text { out }}}{2}
$$

For high flow rate, this approximation is generally representative. However, this assumption may not be valid for the case of a small flow rate and/or long boreholes, where the non-linearity of the temperature profile will be accentuated. For this reason, Marcotte and Pasquier (2008), based on numerical simulations, proposed to replace the mean fluid temperature (Eq. 3) by a so-called $p$-linear average defined as:

$$
T_{f}(t) \approx \frac{p\left(\left|\Delta T_{\text {in }}\right|^{p+1}-\left|\Delta T_{\text {out }}\right|^{p+1}\right)}{(1+p)\left(\left|\Delta T_{\text {in }}\right|^{p}-\left|\Delta T_{\text {out }}\right|^{p}\right)}+T_{o}
$$

Where $\Delta T=T-T_{0}$. They found that using Eq. 3 in the analysis of a TRT can overestimate the borehole resistance whereas using Eq. 4, with $p \rightarrow-1$ gives a better estimate. Beier (2011), using an analytical modeling approach, found that this is indeed the case and that the use of the $p$-linear average, although not exact gives less error for the evaluation of the borehole resistance. Lamarche et al. (2010) confirmed, using numerical simulations, that the borehole resistance deduced when using Eq. 3 is higher than the borehole resistance but closely correspond to the effective borehole resistance. The concept of the effective borehole resistance was introduced by Hellström (1991) and is greater than the borehole resistance because the former takes into account the loss of performance due to the shortcircuiting effect between the two segments of the U-tube inside the borehole. Hellström (1991) found two expressions for the effective resistance given by:

$$
\begin{gathered}
R_{b}^{*}=R_{b} \eta \operatorname{coth}(\eta) \\
\eta=\frac{H}{\dot{m} C_{p} \sqrt{R_{b} R_{a}}}
\end{gathered}
$$

for the case where the borehole temperature is uniform and another equation for the case when the heat flux is uniform along the borehole. In practice, neither assumption is strictly valid but most of the time it gives a good approximation of the internal heat transfer in real boreholes. Eq. 5 involves the expression $R_{a}$ which is called the internal borehole resistance and takes into account the short-circuiting effect between the two legs of the U-tube:

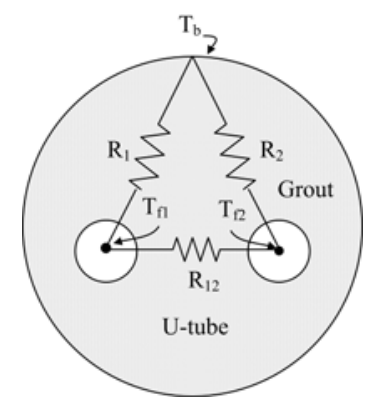

Figure 2 Internal resistance pattern inside a typical borehole 
The borehole resistance $R_{b}$ is found by taking the resistances $R_{1}$ and $R_{2}$ in parallel and the internal resistance is given by evaluating the equivalent resistance between both legs (Fig. 2). In the symmetrical case $\left(R_{1}=R_{2}\right)$, it gives:

$$
R_{b}=\frac{R_{1}}{2} \quad, \quad R_{a}=\frac{4 R_{b} R_{12}}{\left(4 R_{b}+R_{12}\right)}
$$

The concept of effective resistance was extended to double U-tubes arrangements by Zeng et al (2003). In a typical bore field design, just the effective resistance is needed to evaluate the total length of the field. However, if the parameters between the TRT and the real GSHP system vary, like the depth of the borehole, it could be interesting to evaluate both resistances $\left(R_{a}\right.$ and $\left.R_{b}\right)$ in a TRT test to adjust design parameters according to the field response. The following sections give some first results that were found to evaluate these parameters.

\section{MEASUREMENT ON THE TEMPERATURE AT THE BOTTOM}

In order to have a better understanding of the heat transfer inside the borehole, a measurement of the temperature profile is of great interest. Measurement of the temperature profile during TRTs were performed in by Fujii et al. (2009) and Acuña at al. (2011) using fiber optic sensors. Unfortunately, the apparatus to evaluate temperature with optical fiber is expensive. Lamarche et al. (2010) suggested an approach that can be used to evaluate these resistances assuming a prescribed temperature profile inside the borehole. The profile was first derived by Hellström (1991) assuming a thermal exchange between the fluid and a uniform temperature along the borehole. The expressions of the given profile at the bottom and at the exit are given by (Lamarche et al. 2010):

$$
\begin{aligned}
& \theta_{\text {bottom }}=\frac{T_{f, \text { bottom }}(t)-\bar{T}_{b}(t)}{T_{f i}(t)-\bar{T}_{b}(t)}=\cosh (\eta)-\left(\frac{2 \xi \sinh (\eta)}{\cosh (\eta)+\xi \sinh (\eta)} \zeta+\xi\right) \sinh (\eta) \\
& \theta_{\text {out }}=\frac{T_{f o}(t)-\bar{T}_{b}(t)}{T_{f i}(t)-\bar{T}_{b}(t)}=\left(\frac{\cosh (\eta)-\xi \sinh (\eta)}{\cosh (\eta)+\xi \sinh (\eta)}\right)
\end{aligned}
$$

With $\eta$ is given by (6) and

$$
\xi=\frac{L}{\dot{m}_{p} C_{p} 2 R_{b} \eta}, \zeta=\xi \frac{2 R_{b}}{R_{12}}
$$

Having measured value of $\theta_{b o t t o m}$ and $\theta_{o u t}$ Eqs. 8 and 9 can be solved for the two unknown $R_{b}$ and $R_{a}$. They tested their method using numerical simulations while preliminary results in real TRT tests are presented here.

The test was performed at INRS laboratory facilities located in Québec City. The borehole was drilled in $10 \mathrm{~m}$ of unconsolidated till followed by $144 \mathrm{~m}$ of shale from the Sainte-Rosalie Formation in the St. Lawrence Lowlands geological province. A single U-pipe with no space clips was installed in the borehole filled with thermally enhanced grout to make the GHE (Table 1). Heat injection during the TRT was achieved for $81 \mathrm{~h}$ followed by $75 \mathrm{~h}$ of thermal recovery monitoring, where heat injection was stopped but water kept circulating in the GHE. The undisturbed subsurface temperature was measured before the test with a submersible probe lowered in the GHE and was $7.89^{\circ} \mathrm{C}$. Three temperature measurements in the descending pipe leg at depth of $50 \mathrm{~m}, 100 \mathrm{~m}$ and $150 \mathrm{~m}$ were achieved with submersible temperature data loggers during the TRT. The average heat injection rate was $62.7 \mathrm{~W} / \mathrm{m}$, creating a temperature difference of more than $7{ }^{\circ} \mathrm{C}$ between the inlet and outlet of the GHE. At the end of the test the average fluid temperature increased by up to $\sim 20^{\circ} \mathrm{C}$. 
Table 1. GHE configuration and TRT parameters

\begin{tabular}{|c|c|c|c|c|c|c|c|}
\hline $\begin{array}{c}r_{b} \\
(m)\end{array}$ & $\begin{array}{c}L \\
(\mathrm{~m})\end{array}$ & $\begin{array}{c}Q \\
(1 / 5) \\
\end{array}$ & $\begin{array}{c}x_{c} \\
(m) \\
\end{array}$ & $\begin{array}{c}r_{\text {po }} \\
(\mathbf{m})\end{array}$ & $\begin{array}{c}r_{p i} \\
(m)\end{array}$ & $\begin{array}{c}k_{\text {grout }} \\
(\mathrm{W} / \mathrm{mK}) \\
\end{array}$ & $\begin{array}{c}k_{\mathrm{s}} \\
(\mathrm{W} / \mathrm{mK})\end{array}$ \\
\hline 0.057 & 153 & 0.315 & 0.025 & 0.021 & 0.017 & 1.73 & 2.07 \\
\hline
\end{tabular}

In order to solve Eqs. 8 and 9, we need the value of the borehole temperature $T_{b}(t)$. In practice, this is not easy to measure, so here this temperature is estimated using the infinite line source solution (Eq. 1) using the subsurface thermal conductivity found during the TRT. Even though the fluid and the borehole temperature are time dependent, in theory, Eqs 8 and 9, if valid, should be time independent. To verify that, the measured value of the normalized fluid temperature at the bottom and at the exit were plotted as function of time (Fig. 3).

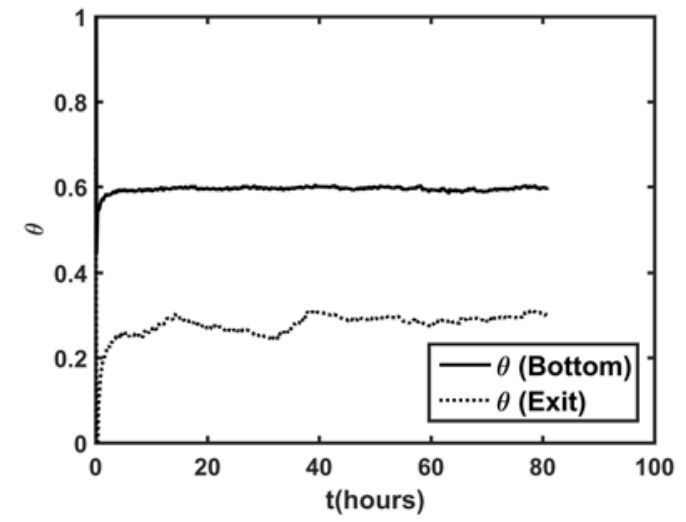

Figure 3 Normalized temperature at the bottom of the borehole and at the exit with respect to time during the heat injection period of the TRT.

The value becomes almost constant after approximately $7 \mathrm{~h}$ which gives a Fourier number of approximately 6, which is a typical value for the validity of steady-flux regime. In our calculations, the mean measured value of the normalized fluid temperature during the steady-flux regime was used in Eq. 8 and 9 in order to find $R_{a}$ and $R_{b}$ (Table 2). Results are compared with the calculated resistances using the multipole method (Claesson and Helltröm 2011). The whole normalized temperature profile is calculated using the calculated resistances (Fig 4). On the same figure, the expected profile, assuming linear profile as it is done usually. In order to have a better idea on the validity of the method, measurements of the fluid temperature in the descending tube at $0 \mathrm{~m}, 50 \mathrm{~m}, 100 \mathrm{~m}$ and $150 \mathrm{~m}$ as well as temperature at $0 \mathrm{~m}$ in the ascending tube are superimposed to the expected normalized temperature in the steady-flux regime. 


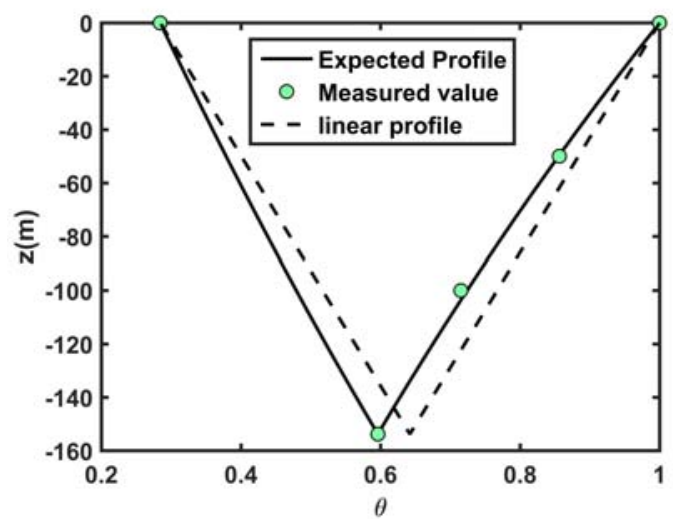

Figure 4 Normalized temperature using Hellstrom profile compared to measured temperature.

\begin{tabular}{cccccc}
\multicolumn{3}{c}{ Measured values } & \multicolumn{2}{c}{ Calculated values (multipole) } \\
\hline \hline $\mathbf{R}_{\mathbf{b}}$ & $\mathbf{R}_{\mathbf{a}}$ & $\mathbf{R}_{\mathbf{b}}{ }^{*}$ & $\mathbf{R}_{\mathbf{b}}$ & $\mathbf{R}_{\mathbf{a}}$ & $\mathbf{R}_{\mathbf{b}} *$ \\
$(\mathbf{m K} / \mathbf{W})$ & $(\mathbf{m K} / \mathbf{W})$ & $(\mathbf{m} \mathbf{K} / \mathbf{W})$ & $(\mathbf{m K} / \mathbf{W})$ & $(\mathbf{m K} / \mathbf{W})$ & $(\mathbf{m K} / \mathbf{W})$ \\
\hline \hline 0.099 & 0.897 & 0.105 & 0.092 & 0.322 & 0.106 \\
\hline
\end{tabular}

\section{Beier Profile}

Beier (2011) proposed a modified equation to calculate the temperature profile inside the U-tube by coupling the borehole resistance network to the undisturbed ground temperature. He latter proposed a modified version taking into account the variation of the ground temperature but, in this manuscript, the uniform temperature case with symmetric configuration $\left(\mathrm{R}_{1}=\mathrm{R}_{2}\right)$ only was considered. The modified temperature profile is normalized with the ground temperature (Fig. 5):

$$
\begin{aligned}
& \theta_{d o w n}(\tilde{z}, t)=\frac{T_{f}(t)-T_{o}}{T_{f i}(t)-T_{o}}=C_{1} \exp \left(a_{1} \tilde{z}\right)+C_{2} \exp \left(a_{2} \tilde{z}\right) \\
& \theta_{u p}(\tilde{z}, t)=\frac{T_{f}(t)-T_{o}}{T_{f i}(t)-T_{o}}=C_{3} \exp \left(a_{3} \tilde{z}\right)+C_{4} \exp \left(a_{4} \tilde{z}\right)
\end{aligned}
$$




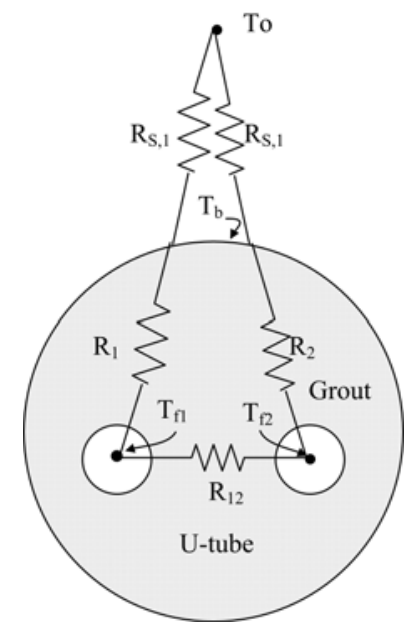

Figure 5 Internal resistance pattern used by Beier

The mathematical expressions for C1, C2, C3, C4, a1, a2, a3 and a4 are described by Beier (2011). They depend on two unknowns $\left(R_{1}\right.$ and $\left.R_{12}\right)$ and known values of the fluid heat capacity, the borehole length and the ground resistance $R_{s, 1}=2 R_{s}$, with $R_{s}$ given by Eq.1. Expressing Eq. 11 and 12 at the bottom and the exit will, in theory, give us the two equations for the two unknowns. One of the advantage of the Beier's profile is that it is not based on a vertically uniform borehole temperature, an assumption has been the subject of debates (Beier 2011, Marcotte and Pasquier 2009). However, one of the disadvantages is that the normalized profile found with Eqs. 11 and 12 is not time independent, even in the steady-flux regime. The short-circuiting effect in the delta equivalent circuit between both legs of the U-tube will follow two possible paths, a direct one through $R_{12}$ and an indirect one via the borehole wall temperature $T_{b}$ (Fig. 2). The equivalent resistance found is the internal resistance $R_{a}$. The resistance $R_{12}$ in Beier's model should then be compared to $R_{a}$ in the delta circuit and not to $R_{12}$.

The equations must be solved with temperature measured at a given time to find the borehole resistance network. This practice can introduce errors since measurements are known to vary randomly and averaged values are always a better approach, when possible. In practice, it was observed that using temperature at different time in solving Eq. 11 and 12, gave large variations in values of $R_{1}$ and $R_{12}$. One could of course average the thermal resistances found. Instead, the approach used here was not to solve Eq. 11 and 12 at a given time but to minimize the least-square error defined by:

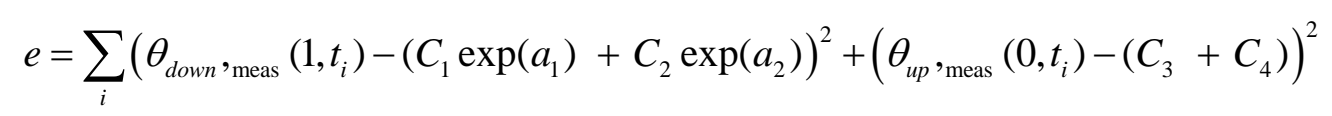

using a Nelder-Mead algorithm. From these thermal resistance results (Table 3), the expected normalized temperature profile given by Eq. 11-12 is compared with the measured temperature values (Fig. 6). As noted previously, normalized fluid temperatures are time dependent and the absolute temperatures are given for a specific time. 


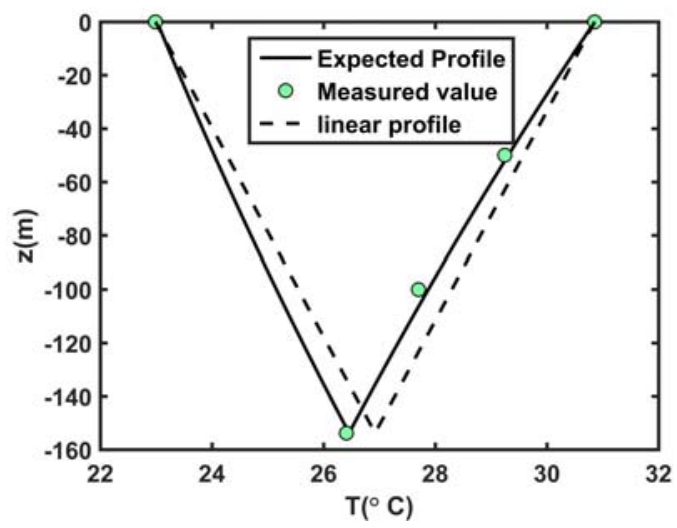

Figure 6 Absolute temperature using Beier's profile compared to measured temperature at $\mathrm{t}=30$ hours.

Table 3

\begin{tabular}{cccc}
\multicolumn{2}{c}{ Measured values } & \multicolumn{2}{c}{$\begin{array}{c}\text { Calculated values } \\
\text { (multipole) }\end{array}$} \\
\hline \hline $\mathbf{R}_{\mathbf{1}}$ & $\mathbf{R}_{\mathbf{1 2}}$ & $\mathbf{R}_{\mathbf{1}}$ & $\mathbf{R}_{\mathbf{1 2}}$ \\
$(\mathbf{m K} / \mathbf{W})$ & $(\mathbf{m K} / \mathbf{W})$ & $(\mathbf{m K} / \mathbf{W})$ & $(\mathbf{m K} / \mathbf{W})$ \\
\hline \hline 0.2 & 6.37 & 0.184 & 2.55 \\
\hline
\end{tabular}

The expected profile matches the experimental data event though the thermal resistances are different (Fig. 6). It is important to note that using resistance in Table 2 with the Beier's profile or the resistance given in Table 3 with the Hellström's profile will give a wrong normalized temperature profile. Comparing Table 2 and 3 , we should remember that $R_{b}$ in the delta model corresponds to $R_{1} / 2$ and $R_{a}$ to $R_{12}$ when the tube placement is symmetric. So, it is observed that the borehole resistance gives very similar final values but the short-circuit resistances show larger variations. It should be remember that during this test the interference was small. Any values of the short-circuit resistances will consequently have a small effect on the final results as long as they are large. Also, it should be remembered that the multi-pole evaluation is based on a symmetric configuration, which is not necessary the case in for real field tests.

\section{DISCUSSION AND CONCLUSIONS}

TRTs are becoming a mature technology for the evaluation of the subsurface thermal conductivity. However, some questions remain concerning the evaluation of the borehole resistance. In this work, preliminary work to find both, the borehole resistance and the internal resistance, using the bottom fluid temperature is presented. It was found that the resistances are dependent of the assumed temperature profile. It is important to note that the profile used should be compatible with the design algorithm to size the bore field when specifying the measured resistances. In this work, we used the profile suggested by Hellström (1991) and Beier (2011). The Beier's method does not need the assumption of a uniform temperature profile. However it brings some conceptual questioning. Indeed, the network approach to represent a typical borehole is based on the quasi steady-state regime (steady-flux) where heat is exchanged between temperatures that vary with time, and where heat transfer can always be expressed by temperature differences divided by some thermal resistances. The thermal flow between both pipes in Beier's network (Fig. 5) will depend on the soil resistances, which are time-dependent. However, this flow is small in a steady-flux regime and $R_{12}$ correspond to the internal resistance $R_{a}$ in the usual network approach. Both approaches used in this study gave satisfying results even though the internal resistance was different than expected theoretically. The main reason is that 
the interference effect was small for the specific test analyzed. In that case, where $\eta$ (Eq. 6) is small, the solution becomes almost independent of $R_{a}$. In future work, field case with higher internal interference effect will be investigated.

\section{ACKNOWLEDGMENTS}

We would like to thank the Natural Sciences and Engineering Research Council of Canada (NSERC) who partly financed this research (RGPIN-2014-06240). Field work was additionally funded with an ENGAGE grant from NSERC in collaboration with Energy Stat.

\section{REFERENCES}

Acuña, J., P. Mogensen and B. Palm. 2011. Distributed Thermal Response Tests on a Multi-Pipe Coaxial Borehole Heat Exchanger. HVAC\&R Research 17(6): 1012-29. doi:10.1080/10789669.2011.625304

Austin W, Yavuzturk C,Spitler J.D. 2000 Development of an in-situ system for measuring ground thermal properties. ASHRAE Trans 2000;106: 365-79.

Beier, R.A.. 2011. Vertical temperature profile in ground heat exchanger during in-situ tests. Renewable Energy 36: 1578-1587.

Carslaw HS, Jaeger JC. 1959. Conduction of heat in solids. 2d ed. New York: Oxford University Press; 1959.

Fujii, H., H. Okubo, K. Nishi, R. Itoi, K. Ohyama and K. Shibata. 2009. An Improved Thermal Response Test for U-Tube Ground Heat Exchanger Based on Optical Fiber Thermometers. Geothermics 38 (4): 399-406. doi:10.1016/j.geothermics.2009.06.002.

Claesson, J, , Hellstrom G. 2011. Multipole method to calculate borehole thermal resistances in a borehole heat exchanger $\gg$. HVAC\&R Research, (17):. 895-911

Hellstrom, G. 1991. Ground Heat Storage. Thermal Analysis of Duct Storage systems. Part I Theory. University of Lund, Department of Mathematical Physics. Lund, Sweden.

Lamarche L, Kajl S, Beauchamp B. 2010 A review of methods to evaluate borehole thermal resistances in geothermal heat-pump systems. Geothermics; 39:187-200

Marcotte, D., Pasquier, P. 2008, On the estimation of thermal resistance in borehole thermal conductivity test., Renewable Energy 33 (11) $2407-2415$.

Raymond, J., G. Robert, R. Therrien and L. Gosselin. 2010. A Novel Thermal Response Test Using Heating Cables. Proceedings of the World Geothermal Congress, Bali, Indonesia: 8p.

Raymond, J., R. Therrien and L. Gosselin. 2011. Borehole Temperature Evolution during Thermal Response Tests. Geothermics 40(1): 69-78. doi:10.1016/i.geothermics.2010.12.002.

Raymond, J., Lamarche. and M-A Blais. 2014. Quality Control Assessment of Vertical Ground Heat Exchangers. ASHRAE Transactions 120(2): SE-14-014.

Spitler, J. D. and S. E.A. Gehlin. 2015. Thermal Response Testing for Ground Source Heat Pump systems-An Historical Review. Renewable and Sustainable Energy Reviews 50: 1125-37. doi:10.1016/j.rser.2015.05.061

Zeng H., Diao N., Fang Z., 2003. Heat transfer analysis of boreholes in vertical ground heat exchangers., International Journal of Heat and Mass Transfer Vol 46 (23), 4467-4481. 